Wander de Pinho Lopes

\title{
Uma Abordagem para Aplicação Integrada de Cenários de Estratégia com Avaliação de Opções Reais em Telecomunicações
}

\author{
Dissertação de Mestrado (Opção profissional)
}

Dissertação apresentada como requisito parcial para obtenção do título de Mestre pelo Programa de PósGraduação em Administração da PUC-Rio.

Orientador: Prof. Walter Lee Ness Jr.

Rio de Janeiro

Março de 2004 
Wander de Pinho Lopes

\title{
Uma Abordagem para Aplicação Integrada de Cenários de Estratégia com Avaliação de Opções Reais em Telecomunicações
}

\begin{abstract}
Dissertação apresentada como requisito parcial para obtenção do título de Mestre pelo Programa de PósGraduação em Administração da PUC-Rio. Aprovada pela Comissão Examinadora abaixo assinada.
\end{abstract}

Prof. Walter Lee Ness Jr. Orientador Departamento de Administração, PUC-Rio

Prof. Antonio Carlos Figueiredo Pinto Departamento de Administração, PUC-Rio

Prof. Eduardo Facó Lemgruber Instituto COPPEAD de Administração, UFRJ

Prof. João Pontes Nogueira Coordenador Setorial do Centro de Ciências Sociais - PUC-Rio 
Todos os direitos reservados. É proibida a reprodução total ou parcial do trabalho sem autorização da universidade, da autora e do orientador.

\section{Wander de Pinho Lopes}

Oito anos de experiência profissional em consultoria na área de gestão financeira, atuando em projetos principalmente na indústria de telecomunicações e utilities (energia e mineração). Sua ênfase é nas áreas de controle gerencial de negócio, planejamento, análise de projetos, orçamento, custos, processos financeiros e benchmarking. É graduado em engenharia mecânica pela UFRJ, com pósgraduação em Administração Financeira pela FGV/RJ.

Ficha Catalográfica

Lopes, Wander de Pinho

Uma abordagem para aplicação integrada de cenários de estratégia com avaliação de opções reais em telecomunicações / Wander de Pinho Lopes ; orientador: Walter Lee Ness Jr. - Rio de Janeiro : PUC, Departamento de Administração, 2004.

165 f. : il. ; $30 \mathrm{~cm}$

Dissertação (mestrado) - Pontifícia Universidade Católica do Rio de Janeiro, Departamento de Administração.

Inclui referências bibliográficas.

1. Administração - Teses. 2. Opções reais. 3. Análise de estratégia. 4. Incerteza (Economia). 5. Finanças. I. Ness Jr., Walter Lee. II. Pontifícia Universidade Católica do Rio de Janeiro. Departamento de Administração. III. Título. 


\section{Agradecimentos}

A Deus, por mais esta conquista.

Aos Professores Walter Ness, meu orientador, e Luiz Brandão, pela inestimável ajuda para a realização deste trabalho.

Ao meu trabalho e à "TPNQM telco", por me ter proporcionado acesso ao rico material utilizado na exemplificação.

À minha família e à Carolina pelo apoio, paciência e compreensão. 


\section{Resumo}

Lopes, Wander de Pinho; Ness, Walter Lee (orientador). Uma Abordagem para Aplicação Integrada de Cenários de Estratégia com Avaliação de Opções Reais em Telecomunicações. Rio de Janeiro, 2004. 165p. Dissertação de Mestrado (Opção profissional) - Departamento de Administração, Pontifícia Universidade Católica do Rio de Janeiro.

Certamente uma das arenas competitivas mais marcada pela incerteza é a indústria de telecomunicações. Características intrínsecas ao negócio, como o alto nível de imobilização e a dispersão geográfica, sob a ação de questões voláteis como demanda, câmbio, tecnologia, regulamentação, dentre outras, podem representar grandes impactos nos resultados do negócio. Esta incerteza sempre existiu, porém nos últimos anos tem estado em maior evidência. Diante desta nova necessidade, os métodos adotados atualmente em planejamento estratégico e em análise de investimentos têm se mostrado pouco eficazes. Não é uma questão de usar uma abordagem melhor ou pior, e sim de usar a mais adequada. Quanto a isto, porém, a única certeza é a de que a melhor forma não é tentar encaixar investimentos e estratégias de uma indústria tão dinâmica como a de telecomunicações em um planejamento estático e rígido.

A proposta deste trabalho é propor e exemplificar um método para descrição e avaliação de estratégia para telecomunicações, baseado no paradigma de opções reais. Este método é uma visão integrada de ferramentas e métodos conhecidos e utilizados em opções reais, análise de cenários e estratégia. A pesquisa não pretende definir o que é melhor para a discussão de análise de estratégias e investimentos em telecomunicações, mas oferecer uma reflexão mais em linha com as peculiaridades do negócio.

\section{Palavras chaves}

opções reais, cenários, incerteza, análise de estratégia, finanças 


\section{Abstract}

Lopes, Wander de Pinho; Ness, Walter Lee (advisor). An Approach for Integrated Application of Strategy Scenario with Real Option Valuation in Telecommunications. Rio de Janeiro, 2004. 165p. Msc Dissertation Departamento de Administração, Pontifícia Universidade Católica do Rio de Janeiro.

Definitely, one of the most uncertain competitive arena in corporate business is the industry of telecommunications. It's intrinsic characteristics, such as high degree of immobilization and geographic dispersion, under the effect of some volatile issues such as demand, currency rate, technology and regulatory affairs, among others, may yield great impacts on business results. This uncertainty has always existed, but in recent times it has been emphasized. In the face of this new necessity, the current methods used in strategic planning and investment analysis have been little effective. It's not a matter of adopting a better or worse approach, but of adopting the most appropriate one. Regarding this, however, the only certainty is that the best alternative is not try to fit investments and strategies of such a dynamic industry as telecommunication in a static and inflexible planning.

The objective of this work is present and illustrates a method for strategy assessment and definition for telecommunications, based on real options. This method is an integrated view of employed and known tools and methods in real options, scenario analysis and strategy. The research does not intend to define what is best for analysis and discussion of strategies and investments in telecommunications, but to offer reflections more in line with the singularities of the business.

\section{Keywords}

real options, scenario, uncertainty, strategy analysis, finance 


\section{Sumário}

1 Introdução 16

1.1. Contextualização 16

1.2. Formulação do Problema 18

1.3. Objetivos 19

1.4. Metodologia 20

1.5. Limitações do Estudo 22

1.6. Relevância do Estudo 23

2 Opções Reais 24

2.1. Apresentação 24

2.2. Histórico 26

2.3. Motivadores para o Uso de Opções Reais 29

2.4. Principais Definições 31

2.4.1. Opções Financeiras 31

2.4.2. Opções Reais 34

2.4.3. Classificações de Opções Reais 41

2.4.4. Uma abordagem Para Configuração de Opções Reais 46

2.4.5. Uma Abordagem Para Análise de Opções Reais 48

2.5. Modelos Matemáticos 51

2.5.1. Funções Estocásticas 53

2.5.2. Árvore Binomial 56

2.5.3. Monte Carlo 61

2.6. Prós e Contras da Aplicação de Opções Reais 63

3 Estratégias Corporativas $\quad 71$

3.1.1. Definição de Estratégia

3.2. Composição de Arenas Competitivas Dinâmicas 74

3.2.1. Ambiente Externo 74

3.2.2. Modelo de Austin (1990) 79

3.2.3. Co-opetition 80

3.2.4. Análise Interna da Organização 82

3.3. Vantagem Competitiva 84

3.4. Alternativas de Estratégias Competitivas 86 
3.4.1. Aprendizagem por Cenários 88

3.4.2. Um Exemplo de Abordagem Para Construção de Cenários 90

4 Alternativas de Estratégia como Opções Reais 94

4.1. Construção de Cenários 96

4.1.1. Definir os principais temas estratégicos 96

4.1.2. Mapear as forças direcionadoras do futuro $\quad 97$

4.1.3. Construir a matriz de cenários $\quad 97$

4.1.4. Elaborar narrativas dos cenários selecionados 98

4.2. Elaboração de Alternativas de Estratégias 99

4.2.1. Mapear dinamismo da arena competitiva 99

4.2.2. Destacar oportunidades e ameaças 100

4.2.3. Levantar evolução necessária dos ativos estratégicos $\quad 100$

4.2.4. Caracterizar as ações estratégicas 100

4.3. Avaliação das Opções Reais 101

4.3.1. Descrever os ativos-objetos em questão 101

4.3.2. Classificar cada Opção Real 102

4.3.3. Calcular o Valor de cada Opção Real 102

4.3.4. Avaliar as Opções Reais 102

5 llustração de uma Aplicação da Abordagem 103

5.1. Ilustração da Construção de Cenários 105

5.1.1. Temas Decisórios $\quad 105$

5.1.2. Forças Direcionadoras do Futuro 108

5.1.3. Matriz de Cenário 110

5.1.4. Narrativas dos Cenários 112

5.2. llustração da Elaboração de Alternativas Estratégicas $\quad 115$

5.2.1. Elaboração de Alternativas Estratégicas 115

5.2.2. Evolução dos Ativos Estratégicos 117

5.2.3. Ações Estratégicas Atreladas aos Ativos Estratégicos $\quad 117$

5.3. Ilustração da Avaliação das Opções Reais 119

5.3.1. Classificação das Opções Reais 126

5.3.2. Valores e Avaliação das Opções Reais $\quad 127$

6 Conclusões 137

7 Referências Bibliográficas $\quad 144$ 
8 Anexos

8.1. Relatórios de Frequência - Simulação de Monte Carlo

8.2. Modelo com Flexibilidade - Árvore de Decisão

8.3. Modelo com Flexibilidade - Árvore de Decisão com Opção de Expansão 


\section{Lista de figuras}

Figura 1: Valor de uma opção e sua volatilidade, segundo Ross (1995), p. 447

Figura 2: Valor de opção de cancelamento. Exemplo retirado de Copeland \& Keenan (1998, n.2.), p. 42.

Figura 3: Identificação de uma opção real 40

Figura 4: Exemplo de tipologia para classificação de opções reais 44

Figura 5: Processo de simulação de Monte Carlo segundo

Copeland \& Antikarov (2001), p. 245.

Figura 6: Matriz de decisão para opções reais, proposta por Luehrman (1998, n²), p. 93.

Figura 7: Movimentos de subida e de descida de árvore binomial 56

Figura 8: Ilustração do segundo passo de uma árvore binomial 59

Figura 9: Ilustração de uma árvore binomial expandida 61

Figura 10: Mapeamento das possíveis flutuações em um projeto, proposto por Amram \& Kulatilaka (1999), p. 101.66

Figura 11: Comparação entre métodos de avaliação de projetos, por Copeland \& Keenan (1998, n.2), p. 45.

Figura 12: Limites entre arenas competitivas cada vez mais obscuros 74

Figura 13: Modelo de Porter 75

Figura 14: Análise de alternativas estratégicas como opções reais 94

Figura 15: Análise de alternativas estratégicas como opções reais - Construção de Cenários

Figura 16: Análise de alternativas estratégicas como opções reais - Elaboração de Alternativas Estratégicas

Figura 17: Análise de alternativas estratégicas como opções reais - Avaliação das Opções Reais

Figura 18: Iniciativas Estratégicas

Figura 19: Option space dos projetos da TPNQM telco 121

Figura 20: Condições da árvore de decisão 132

Figura 21: Definição da árvore de decisão, para o software DPL 133

Figura 22: Condições da árvore de decisão com a opção 
de expansão

Figura 23: Definição da árvore de decisão com a opção de expansão, para o software DPL 


\section{Lista de tabelas}

Tabela 1: Avaliação preliminar das opções reais 120

Tabela 2: Dados do fluxo de caixa do projeto selecionado 122

Tabela 3: Dados do financiamento do projeto selecionado 122

Tabela 4: Curva de taxa LIBOR 123

Tabela 5: Dados da amortização do financiamento 123

Tabela 6: Montantes considerados para o fluxo operacional do projeto escolhido 124

Tabela 7: Fluxo de caixa sem flexibilidade do projeto selecionado 125

Tabela 8: Dados da opção de expansão 127

Tabela 9: Resultados das simulações de Monte Carlo 130 


\section{Lista de quadros}

Quadro 1: Exemplo de opção de compra 32

Quadro 2: Exemplo de opção de venda 32

Quadro 4: Típicas situações geradoras de opções reais, por setor. 39

Quadro 5: Caracterização do ambiente externo segundo Austin (1990) 80

Quadro 6: Temas de decisão 108

Quadro 7: Caracterização das forças direcionadoras 109

Quadro 8: Padrão de comportamento das forças direcionadoras 110

Quadro 9: Matriz de cenário 111

Quadro 10: Eventos do cenário selecionado 113

Quadro 11: Dinâmica do cenário selecionado 113

Quadro 12: Resumo das conclusões sobe cada objetivo proposto 116

Quadro 13: Resumo dos recursos e capacidades a serem criados e/ou consolidados 117

Quadro 14: Descrição das incertezas associadas ao projeto 128

Quadro 15: Resumo das conclusões sobe cada objetivo proposto 138 


\section{Siglas e Abreviaturas}

ANATEL : Agência nacional de Telecomunicações

ASP: Application Service Provider. Serviço de hospedagem e helpdesk de aplicativos, dentre outros.

B2B: Business to Business

CAPEX: CAPital EXenditure

CNS: Corporate Network Services

CST : Companhia Siderúrgica de Tubarão

CVRD : Companhia Vale do Rio Doce

Downstream: negócio de distribuição de refinados de petróleo

EAI: Enterprise Application Integration

EBITDA: Earning Before Incoming Taxes, Depreciation and Amortization

EVA: Economic Value Added (valor econômico adicionado)

IAP: Internet Access Provider

IRR: Internal Rate of Return (taxa interna de retorno)

LAIR: Lucro Antes do Imposto de Renda

LAN: Local Area Network

LDI: Longa Distância Internacional

LDN: Longa Distância Nacional

MVNO: Mobile Virtual Network Operator

NGN: Next Generation Network - representa a unificação em uma infra-estrutura única das redes comutada fixa, IP e móvel. A idéia é prover foco na customização dos serviços de comunicação ao nível de assinante, tanto no mercado corporativo quanto no residencial.

NPV: Net Present Value (VPL - valor presente líquido)

Pixel: unidade básica de uma cor programável na tela de um computador.

O tamanho físico de um pixel depende da resolução da tela (é uma palavra inventada do "elemento de foto")

PME: pequenas e médias empresas

QoS: Quality of Service

SLA: Service Level Agreement 
SMP: Serviço Móvel Pessoal

SOHO: Small Office and Home Office

STFC: Sistema de Telefonia Fixa Comutada

Upstream: negócio de exploração e refino de petróleo

VoD: Video on Demand

WACC: Weighted-Average Cost of Capital

W-LAN: Wide - Local Access Network 